\title{
The topological structure of supergravity: an application to supersymmetric localization
}

\author{
Camillo Imbimbo ${ }^{a, b}$ and Dario Rosa ${ }^{c}$ \\ ${ }^{a}$ Dipartimento di Fisica, Università di Genova, \\ Via Dodecaneso 33, Genoa 16146, Italy \\ ${ }^{b}$ INFN, Sezione di Genova, \\ Via Dodecaneso 33, Genoa 16146, Italy \\ ${ }^{c}$ School of Physics, Korea Institute for Advanced Study (KIAS), \\ Seoul 02455, Korea \\ E-mail: camillo.imbimbo@ge.infn.it, Dario85@kias.re.kr
}

ABSTRACT: The BRST algebra of supergravity is characterized by two different bilinears of the commuting supersymmetry ghosts: a vector $\gamma^{\mu}$ and a scalar $\phi$, the latter valued in the Yang-Mills Lie algebra. We observe that under BRST transformations $\gamma$ and $\phi$ transform as the superghosts of, respectively, topological gravity and topological Yang-Mills coupled to topological gravity. This topological structure sitting inside any supergravity leads to universal equivariant cohomological equations for the curvatures 2-forms which hold on supersymmetric bosonic backgrounds. Additional equivariant cohomological equations can be derived for supersymmetric backgrounds of supergravities for which certain gauge invariant scalar bilinears of the commuting ghosts exist. Among those, $N=(2,2)$ in $d=2$, which we discuss in detail in this paper, and $N=2$ in $d=4$.

KeYwords: BRST Quantization, Supergravity Models, Supersymmetric Gauge Theory, Topological Field Theories

ArXiv EPRINT: 1801.04940 


\section{Contents}

1 Introduction 1

2 The BRST formulation of supergravity 5

$\begin{array}{llll}3 & \text { Topological Yang-Mills coupled to topological gravity } & 7\end{array}$

$\begin{array}{lll}4 & \text { The topological structure of supergravity } & 9\end{array}$

5 The universal cohomological equations for supersymmetric backgrounds 13

$6 \quad$ The gauge invariant ghost bilinears 15

7 An example: $d=2 N=(2,2)$ supergravity 17

8 Conclusions $\quad 19$

A Equivariant cohomology in dimension $2 \quad 22$

\section{Introduction}

Localization has proven to be a powerful tool to study supersymmetric quantum field theories (SQFT) on curved backgrounds. ${ }^{1}$ To identify localizable backgrounds one couples supersymmetric matter field theories to classical supergravity: setting the supersymmetry variations of the fermionic supergravity fields - both gravitinos and gauginos - to zero, one obtains equations for the local supersymmetry spinorial parameters, a strategy first exploited in [2]. These differential equations, that are often named generalized Killing spinor equations, admit non-trivial solutions only for special configurations of the bosonic fields of the supergravity multiplet. The relevant supergravity and the particular generalized Killing spinor equations depend on the global symmetries of the specific SQFT one is interested in: indeed, conserved currents of the SQFT that one would like to probe couple to gauge fields which must sit in supergravity multiplets.

It was observed in [3] and [4] that the generalized Killing spinor equations for certain extended supergravities in 3 and 2 dimensions are equivalent to cohomological equations which are obtained by setting to zero the BRST variations of fermionic fields of topological gravity coupled to certain topological gauge systems. In this paper we will provide a conceptual explanation of the equivalence between the generalized Killing spinor equations of supergravity and the cohomological equations derived from topological gravity.

\footnotetext{
${ }^{1}$ The literature on supersymmetric localization is enormous. See [1] for an extensive overview of the main recent results.
} 
Our starting point will be the BRST formulation of supergravity. We will revisit this in section 2. The BRST structure of a given supergravity theory is specified by the number of its local supersymmetries and by its bosonic local gauge symmetries. For each local symmetry one introduces ghost fields - anti-commuting for bosonic local symmetries and commuting for fermionic ones. The bosonic local gauge symmetries always include at least local reparametrizations and local Lorentz transformations: on top of those one can consider additional Yang-Mills local symmetries. These may be associated, for example, to global R-symmetries of the matter SQFT to which supergravity can be coupled.

We will show that the BRST algebra of any supergravity theory takes the form

$$
S^{2}=\mathcal{L}_{\gamma}+\delta_{i_{\gamma}(A)+\phi}
$$

Here $S$ is obtained from the nilpotent BRST operator $s$ by subtracting the transformations associated to the bosonic gauge symmetries; $\mathcal{L}_{\gamma}$ is the Lie derivative along the vector field $\gamma^{\mu}$ of ghost number 2

$$
\gamma^{\mu}=-\frac{1}{2} \sum_{i} \bar{\zeta}^{i} \Gamma^{\mu} \zeta^{i}
$$

where $\zeta^{i}$, with $i=1, \ldots, N$, are the commuting supersymmetry Majorana ghosts ${ }^{2}$ and $\Gamma^{\mu}$, with $\mu=1, \ldots, d$, are the Dirac matrices in curved space-time of dimension $d ; \delta_{c}$ denotes a gauge transformation with parameter $c$ and $i_{\gamma}(A)$ is the contraction of the YM gauge field $A$, belonging in the supergravity multiplet, with the vector field $\gamma^{\mu}$; the scalar field $\phi$ lives in the adjoint representation of the bosonic YM gauge symmetry group. $\phi$, like $\gamma^{\mu}$, is a combination of ghost number 2 of the supergravity fields, bilinear in the commuting ghosts $\zeta^{i}$ and it is independent of the other ghost fields.

The vector ghost bilinear $\gamma^{\mu}$ and the adjoint-valued scalar ghost bilinear $\phi$ completely specify the supergravity BRST algebra - and thus the supergravity model. It should be remarked that the vector ghost bilinear $\gamma^{\mu}$ has, for any supergravity, the universal form (1.2); on the other hand the dependence of the adjoint-valued scalar ghost bilinear $\phi$ on the bosonic fields is model dependent. We will provide explicit expressions for $\phi$ for the supergravity theories that we discuss in this paper.

The algebra (1.1) encodes the connection between supergravity and topological theories: indeed both the field $\gamma^{\mu}$ and the field $\phi$ admit a natural topological interpretation on which we will elaborate in section 4 . We will show that, under supergravity BRST transformations, the ghost number 2 vector $\gamma^{\mu}$ transforms precisely as the superghost of topological gravity while $\phi$ transforms as the superghost of a topological Yang-Mills multiplet. Explicitly, the supergravity BRST operator $S$ acts on $\phi$ as follows

$$
\begin{aligned}
S \phi & =i_{\gamma}(\lambda) \\
S \lambda & =i_{\gamma}(F)-D \phi \\
S F & =-D \lambda
\end{aligned}
$$

\footnotetext{
${ }^{2}$ For concreteness, we are taking the commuting ghosts to be Majorana spinors in Minkowski space-time here and in the rest of the paper, with the exception of section 7 . We take the Minkowski signature to be $(+,-, \ldots,-)$. When $N$ is even it might be more convenient to work with Dirac spinors. When the space-time dimension permits, one could adapt the discussion to Euclidean signature: this is what we do in section 7 where we describe the application to localization of $N=(2,2)$ supergravity in two dimensions.
} 
where $F$ is the field strength 2 -form associated to bosonic Yang-Mills symmetries and $\lambda$ is a ghost number 1 combination of supergravity fields which we will call the (composite) "topological gaugino". $\lambda$ is defined by the supergravity BRST variation of the gauge field

$$
S A=\lambda
$$

The BRST transformations (1.3) are identical to the BRST rules of the topological YangMills multiplet $(F, \lambda, \phi)$ coupled to topological gravity, once one identifies the supergravity bilinear $\gamma^{\mu}$ with the topological gravity superghost. The topological gravity BRST transformations read indeed

$$
\begin{aligned}
S g_{\mu \nu} & =\psi_{\mu \nu} \\
S \psi_{\mu \nu} & =\mathcal{L}_{\gamma} g_{\mu \nu} \\
S \gamma^{\mu} & =0
\end{aligned}
$$

where $g_{\mu \nu}$ is the metric and $\psi_{\mu \nu}$ is the topological gravitino. The consistency of (1.5) with (1.3) hinges on the supergravity transformations for the supersymmetry ghosts and vierbein

$$
\begin{aligned}
& S \zeta^{i}=i_{\gamma}\left(\psi^{i}\right) \\
& S e^{a}=\sum_{i} \bar{\zeta}^{i} \Gamma^{a} \psi^{i}
\end{aligned}
$$

where $\psi^{i}$ are the gravitinos and $e^{a}=e_{\mu}^{a} d x^{\mu}$ the vierbein 1-forms. Note that the supergravity BRST rules (1.6) are universal, i.e. independent of the specific supergravity theory. The fact that the supergravity transformations (1.6) imply, in particular, the invariance of the bilinear (1.2) was observed first in [5].

In section 3 we will review the coupling, in generic dimensions, of topological gravity to topological YM in any dimensions. This was worked out in [6] and [3] in a three-dimensional context. It is well-known that the BRST operator of "rigid" topological YM is geometrically the de Rham differential on the space $\mathcal{A}$ of gauge connections, equivariant with respect to gauge transformations. Analogously, the BRST operator of topological gravity in (1.5) is the de Rham differential on the space Met of metrics, equivariant with respect to spacetime diffeomorphisms. The BRST operator $S$ in (1.3), which will be discussed in section 3, is instead the de Rham differential on the space $\mathcal{A} \times$ Met, equivariant with respect to both diffeomorphisms and gauge transformations. Independently of the application to supergravity that we consider in this work, this operator should have applications to the study of metric dependence of Donaldson invariants.

In the rest of this paper we apply to supersymmetric localization the topological structure that we have discovered sitting inside the supergravity BRST algebra. Supersymmetric bosonic backgrounds are obtained by setting to zero the supergravity BRST variations of all the fermionic supergravity fields. We will refer to the set of such backgrounds as the localization locus. On the localization locus also the supergravity BRST variations of the composite fields $\lambda=S A$ and $\psi_{\mu \nu}=S g_{\mu \nu}$, must vanish. The resulting equations will be 
analyzed in section 5. By imposing that the supergravity BRST variation of the topological gravitino vanishes one gets the equation

$$
S \psi_{\mu \nu}=\mathcal{L}_{\gamma} g_{\mu \nu}=D_{\mu} \gamma_{\nu}+D_{\nu} \gamma_{\mu}=0
$$

which expresses the request that the vector bilinear $\gamma^{\mu}$ be an isometry of the space-time metric $g_{\mu \nu}$. The fact that, on the localization locus, the vector bilinear $\gamma^{\mu}$ must be a Killing vector of the space-time metric is well-known in the supergravity literature. The vanishing of the supergravity BRST variation of the topological gaugino gives instead the equation

$$
D \phi-i_{\gamma}(F)=0
$$

This equation is universal, in the sense that it is valid, on the localization locus, for any supergravity in any dimensions. It appears to be a novel equation which has not been yet explored in either supergravity or topological field theory literature.

Eq. (1.8) takes values in the adjoint representation of the total bosonic YM gauge group: it splits therefore into an equation valued in the Lorentz local algebra and one in the additional YM symmetry algebra. When either of these are non-abelian, the universal localization equation (1.8) is non-linear. In this case, although equation (1.8) has topological roots, it does not directly defines a cohomological problem. To connect eq. (1.8) to cohomology theory, we need to extract its gauge invariant content. To this end, let us define the generalized Chern classes

$$
\mathbb{C}_{n}=\operatorname{Tr}(F+\phi)^{n}=\operatorname{Tr} F^{n}+\cdots+\operatorname{Tr} \phi^{n}
$$

which are gauge invariant polyforms. When the backgrounds $F$ and $\phi$ satisfy the localization equation (1.8) the generalized Chern classes obey the equation

$$
\mathcal{D}_{\gamma} \mathbb{C}_{n}=\left(d-i_{\gamma}\right) \mathbb{C}_{n}=0
$$

The differential $\mathcal{D}_{\gamma} \equiv d-i_{\gamma}$ is the coboundary operator defining the de Rham cohomology of forms on space-time, equivariant with respect to the action associated to the Killing vector $\gamma^{\mu}$. We will refer to this cohomology as the $\gamma$-equivariant cohomology. Eq. (1.10) says therefore that the generalized Chern classes $\mathbb{C}_{n}$ are the $\gamma$-equivariant extensions of the ordinary Chern classes $c_{n}=\operatorname{Tr} F^{n}$.

The ordinary Chern classes $c_{n}$ are integer-valued when $F$ is the curvature of an hermitian connection. We will compute explicitly the equivariant extensions of these classes for $N=(2,2)$ supergravity in $d=2$. It will turn out that the equivariant extensions defined by this supergravity are integer-valued as well. We believe this integrality property is a general feature of supersymmetric bosonic backgrounds, although we have not yet a proof of this. At any rate, different values of the $\gamma$-equivariant classes (1.10) label different branches of the localization locus. On each of these branches moduli spaces of inequivalent localizing backgrounds may exist - all with the same (integral) values of the equivariant Chern classes.

Eqs. (1.7), (1.8) and its consequence (1.10) are obtained by putting to zero the BRST variation of specific (composite) fermions: the topological gravitino and gauginos, belonging 
in topological multiplets whose ghost number 0 components are (respectively) the metric and the YM curvature. It should be stressed that these equations do not, in general, completely characterize the localization locus. One can obtain additional, independent, gauge invariant cohomological equations for bosonic supersymmetric backgrounds by setting to zero the variations of other, independent, gauge invariant composite fermions. In section 6 we will discuss how to construct gauge invariant composite fermions out of the (universal) sector of supergravity which does not involve the auxiliary fields. These fermions sit in topological gauge invariant multiplets whose ghost number 2 scalars involve only the supergravity ghosts $\zeta^{i}$. The 2 -form components of these multiplets depend on the auxiliary fields. They are not, in general, curvature of gauge fields associated to local symmetries. To determine them one needs the knowledge of the full off-shell supergravity BRST rules.

Multiplets of this kind, that we will call gauge invariant topological multiplets, can be constructed only for specific supergravity theories with suitable YM local gauge invariance. In section 6 we will find and discuss examples of these multiplets for $d=2 N=(2,2)$ supergravity and $d=4 N=2$ supergravity with $\mathrm{SU}(2) \mathrm{YM}$ gauge symmetry.

The localization equations associated to the gauge invariant topological multiplets also take the form of $\gamma$-equivariant cohomology equations for classes of degree 2 . These equations are very powerful. They do not appear to have the integrality properties of the cohomology equations associated to the curvature topological multiplets. Their continuous moduli spaces parametrize the inequivalent localizing backgrounds within a given topological branch classified by the equivariant Chern classes in the equation (1.10). We will verify this explicitly for $N=(2,2) d=2$ supergravity in section 7 , where we will show that the curvature topological multiplet for this theory can be expressed on the localization locus as a quadratic function of the gauge invariant topological multiplets.

We postpone to future work an analysis of the localization equations associated to the gauge invariant topological multiplets of $N=2$ supergravity with $\mathrm{SU}(2) \mathrm{YM}$ gauge symmetry in four dimensions. A complete study of these equations requires the knowledge of the off-shell BRST transformations for this model. We believe that such a study might lead to the solution of the long standing problem of the classification of localizing backgrounds for $N=2 d=4$ supergravity.

\section{The BRST formulation of supergravity}

In the BRST framework one introduces ghost fields of ghost number +1 in correspondence to each of the local symmetries. The bosonic local symmetries of supergravity are diffeomorphisms and YM gauge symmetries. We will denote by $\xi^{\mu}$ the anti-commuting vector ghost field associated to diffeomorphisms, and by $c$ the anti-commuting ghost associated to the YM gauge symmetry which takes values in the adjoint representation of the YM algebra. The YM gauge symmetries always include local Lorentz transformations. Beyond local Lorentz gauge symmetry, we will also allow for additional YM gauge symmetries. For the application to localization, for example, these additional YM gauge symmetries include the R-symmetries of the supersymmetric quantum field theory whose coupling to supergravity one is considering. 
In correspondence with the $N$ local supersymmetries, one introduces commuting supergravity spinorial Majorana ghosts $\zeta^{i}$, with $i=1, \ldots N$, whose BRST transformation rules have the form

$$
s \zeta^{i}=i_{\gamma}\left(\psi^{i}\right)+\text { diffeos }+ \text { gauge transfs }
$$

In this equation $s$ is the nilpotent BRST operator

$$
s^{2}=0
$$

$\psi^{i}=\psi_{\mu}^{i} d x^{\mu}$ are the Majorana gravitinos and $\gamma^{\mu}$ is the following vector bilinear of the commuting ghosts ${ }^{3}$

$$
\gamma^{\mu} \equiv-\frac{1}{2} \sum_{i} \bar{\zeta}^{i} \Gamma^{a} \zeta^{i} e_{a}^{\mu}
$$

where $e_{a}^{\mu}$ are the inverse of the vierbein $e^{a} \equiv e_{\mu}^{a} d x^{\mu}$. The vector $\gamma^{\mu}$ has ghost number +2 .

Both the Majorana ghosts $\zeta^{i}$ and gravitinos $\psi^{i}$ carry a label $i=1, \ldots N$ on which the $O(N)$ subgroup of the R-symmetry group acts. The full R-symmetry group can however be as large as $\mathrm{U}(N)$.

The BRST transformations of the vierbein are universal

$$
s e^{a}=\sum_{i} \bar{\psi}^{i} \Gamma^{a} \zeta^{i}+\text { diffeos }+ \text { gauge transfs }
$$

We will denote the action of diffeomorphisms with $\mathcal{L}_{\xi}$, the Lie derivative associated with the vector field $\xi^{\mu}$. The BRST transformations of the diffeomorphism ghost are

$$
s \xi^{\mu}=-\frac{1}{2} \mathcal{L}_{\xi} \xi^{\mu}-\frac{1}{2} \sum_{i} \bar{\zeta}^{i} \Gamma^{a} \zeta^{i} e_{a}^{\mu}
$$

It was noted in [5] that the BRST transformations (2.1) and (2.4) imply that the vector ghost bilinear $\gamma^{\mu}$ transforms as follows

$$
s \gamma^{\mu}=-\mathcal{L}_{\xi} \gamma^{\mu}
$$

This transformation law coincides precisely with the BRST transformation rule for the superghost of topological gravity [7]. Indeed, the full BRST transformations of topological gravity write

$$
\begin{aligned}
s \gamma^{\mu} & =-\mathcal{L}_{\xi} \gamma^{\mu} \\
s g_{\mu \nu} & =-\mathcal{L}_{\xi} g_{\mu \nu}+\psi_{\mu \nu}, \\
s \xi^{\mu} & =-\frac{1}{2} \mathcal{L}_{\xi} \xi^{\mu}+\gamma^{\mu} \\
s \psi_{\mu \nu} & =-\mathcal{L}_{\xi} \psi_{\mu \nu}+\mathcal{L}_{\gamma} g_{\mu \nu}
\end{aligned}
$$

where $g_{\mu \nu}$ is the metric, $\psi_{\mu \nu}$ is the topological gravitino and $\gamma^{\mu}$ the vector superghost. From this it is apparent that also the supergravity BRST transformation rules (2.5) match the topological gravity ones, once the topological gravity superghost is identified with the supergravity ghost bilinear $\gamma^{\mu}$ according to (2.3). The formal coincidence of the topological relations (2.7) with the supergravity ones (2.5), (2.6) is the first hint that a topological sector hides behind the supergravity BRST rules. In the following we will bear this to light.

\footnotetext{
${ }^{3}$ To avoid confusions, we will denote with $\gamma^{\mu}$ the ghost bilinear and with $\Gamma^{a}$ the Dirac matrices.
} 


\section{Topological Yang-Mills coupled to topological gravity}

We have seen in the previous section that some of the supergravity BRST transformation rules take a form which is identical to the BRST transformations of topological gravity. In order to uncover the full topological content of the supergravity BRST rules it is necessary to discuss the coupling of topological gravity to topological Yang-Mills gauge theories. This was derived, in the form we present here, in [6] and [3] in the specific context of 3-dimensional gauge theories. ${ }^{4}$ In this section we will describe this construction in generic dimension and explain its geometric meaning. ${ }^{5}$

The coupling of topological gravity to topological Yang-Mills can be useful in different contexts. The first one is when both topological gravity and Yang-Mills fields are dynamical. This gravitational theory is relevant to study the cohomology of the space of space-time metrics. One could also consider the situation in which only the YM degrees of freedom are dynamical while the topological gravity fields play the role of classical backgrounds. In this case the coupling to topological gravity probe the dependence of the quantum physical correlators of the YM theory on the background metric. It might be a useful tool to study, among other things, the occurence of possibile quantum anomalies of the classical topological invariance [6] or wall-crossing phenomena in Donaldson theory [12-14].

In the following sections topological YM and topological gravity fields will appear as composites of the "microscopic" supergravity fields. In section 4 we will explain how such composites emerge from the BRST formulation of supergravity: we will use the existence of this topological structure inside supergravity to derive general information regarding localization of supersymmetric gauge theories coupled to classical supergravity. In this section - and only in this section — we think, instead, of topological YM and topological gravity as fundamental "microscopic" theories.

The fields of the topological Yang-Mills theory include, beyond the gauge connection $A=A_{\mu}^{a} T^{a} d x^{\mu}$, the topological gaugino $\lambda=\lambda_{\mu}^{a} T^{a} d x^{\mu}$ of ghost number +1 , the gauge ghost $c=c^{a} T^{a}$ of ghost number +1 and the super-ghost $\phi=\phi^{a} T^{a}$ of ghost number $+2 .^{6}$

The BRST rules of "rigid" topological YM - i.e. topological YM before coupling it to topological gravity — read

$$
\begin{aligned}
s c & =-\frac{1}{2}[c, c]+\phi \\
s A & =-D c+\lambda \\
s \lambda & =-D \phi-[c, \lambda] \\
s \phi & =-[c, \phi]
\end{aligned}
$$

\footnotetext{
${ }^{4}$ BRST transformations for topological gravity in $d=4$ coupled to abelian gauge theory were written also in [8]. Although those BRST rules and the one we are presenting in this section are related by a field redefinition, we note that this field redefinition is essential to obtain gauge covariant BRST rules, and, thus, gauge covariant localization equations.

${ }^{5} \mathrm{~A}$ generalization of this construction in $d=2$ has been considered in [9], where the coupling of topological gravity to 2-dimensional Poisson sigma models $[10,11]$ has been worked out.

${ }^{6}$ The matrices $T^{a}$, with the index $a$ running over the adjoint representation of the gauge group, are usually taken to be the generators of the Lie algebra of the gauge group in the fundamental representation.
} 
It is convenient to introduce an operator $S$, whose action is defined on all the fields but not on the ghost field $c . S$ is related to the nilpotent $s$ by the relation

$$
S \equiv s+\delta_{c}
$$

where $\delta_{c}$ denotes the gauge transformation with parameter $c$. We will refer to $S$ as the BRST operator equivariant with respect to local gauge transformations. The nilpotency of $s$ is equivalent to the algebra

$$
S^{2}=\delta_{\phi}
$$

It is well known that the operator $S$ defined in (3.1) and (3.2) has to be interpreted as the de Rham differential on the space $\mathcal{A}$ of gauge connections, equivariant with respect to local gauge transformations. Hence, the BRST cohomology of $S$ corresponds, geometrically, to the cohomology of $\mathcal{A}$ modulo local gauge transformations.

Coupling topological YM to topological gravity means to find an extension of the nilpotent BRST operator $s$ which includes local diffeomorphism transformations, with a ghost parameter $\xi^{\mu}$ that transforms according to the BRST rules of topological gravity (2.7). The request of nilpotency of $s$ dictates the following deformation of (3.1)

$$
\begin{aligned}
S A & =\lambda \\
S \lambda & =i_{\gamma}(F)-D \phi \\
S \phi & =i_{\gamma}(\lambda)
\end{aligned}
$$

where $F=d A+A^{2}$ is the gauge field strength 2-form, $i_{\gamma}$ denotes the contraction of a form with the vector field $\gamma^{\mu}$ and $S$ is equivariant both with respect to local gauge transformations and to diffeomorphisms

$$
S=s+\delta_{c}+\mathcal{L}_{\xi}
$$

The new equivariant $S$ of topological YM coupled to topological gravity satisfies the relation

$$
S^{2}=\delta_{\phi+i_{\gamma}(A)}+\mathcal{L}_{\gamma}
$$

The BRST transformations of the gauge ghost $c$ are

$$
s c=-\frac{1}{2}[c, c]-\mathcal{L}_{\xi} c+\phi+i_{\gamma}(A)
$$

The equivariant $S$ defined in (3.4)-(3.5) is the de Rham differential on the product space $\mathcal{A} \times$ Met, where Met is the space of space-time metrics, equivariant with respect to the action of both local gauge transformations and diffeomorphisms.

We can re-cast the first equation of (3.4) and the BRST variation of the gauge ghost (3.7) in terms of a curvature $\mathbb{F}$ of the superconnection $c+A$

$$
\delta(c+A)+(c+A)^{2}=F+\lambda+\phi \equiv \mathbb{F}
$$

where

$$
\delta=s+d+\mathcal{L}_{\xi}-i_{\gamma}
$$


is nilpotent

$$
\delta^{2}=0
$$

The rest of the transformations (3.4) are equivalent to the Bianchi identity

$$
\delta \mathbb{F}+[c+A, \mathbb{F}]=0
$$

Therefore the gauge invariant generalized polynomials

$$
c_{n}(\mathbb{F})=\operatorname{Tr} \mathbb{F}^{n}
$$

are $\delta$-invariant

$$
\left(S+d-i_{\gamma}\right) c_{n}(\mathbb{F})=0
$$

In the context in which both YM and gravity are dynamical, eq. (3.13) expresses the fact that the generalized forms $c_{n}(\mathbb{F})$ encode observables of the theory constructed purely in terms of YM degrees of freedom. In the context in which only the YM degrees of freedom are dynamical, eq. (3.13) gives rise to a (classical) Ward identity which controls the dependence of quantum correlators of the observables associated to $c_{n}(\mathbb{F})$ on the background metric. In the supergravity context eq. (3.13) implies - as we will show in the following sections - cohomological differential equations which are satisfied by localizable backgrounds of classical supergravity.

\section{The topological structure of supergravity}

The BRST formulation of supergravity involves both fields with zero ghost number and ghost fields. As recalled in section 2, one must introduce both anti-commuting ghost fields - which we denoted with $\xi$ and $c$ - associated with bosonic gauge invariance and commuting ghost fields $\zeta^{i}$ associated with local supersymmetry. In the rest of this section we will refer, somewhat unorthodoxly, both to zero ghost number fields and to commuting ghosts $\zeta^{i}$ as "matter fields". We will denote them collectively by $M$.

When acting on matter fields $M$ the nilpotent BRST operator $s$ has the form

$$
s M=-\mathcal{L}_{\xi} M-\delta_{c} M+\hat{M}(M)
$$

Here $\delta_{c} M$ denotes the gauge group action on $M$ with parameter $c$. Eq. (4.1) defines therefore the composite field $\hat{M}(M)$, which is in general a function of the matter fields of the theory - but not of the anti-commuting ghosts $\xi$ and $c$. For example, from the BRST rules (2.1), we deduce that

$$
\hat{\zeta}=i_{\gamma}(\psi)
$$

The BRST transformation rules of the bosonic ghost associated to the gauge symmetries have a slightly different structure

$$
\begin{aligned}
s \xi^{\mu} & =-\frac{1}{2} \mathcal{L}_{\xi} \xi^{\mu}+\gamma^{\mu} \\
s c & =-\frac{1}{2}[c, c]-\mathcal{L}_{\xi} c+\hat{c}
\end{aligned}
$$


Here $\gamma^{\mu} \equiv \hat{\xi}^{\mu}$ is the universal bilinear defined in (2.3). The second equation defines instead $\hat{c}$ which is a function of ghost number 2 of the matter fields: its specific form characterizes the particular supergravity we are considering. By imposing nilpotency of $s$ on $M$

$$
0=s^{2} M
$$

and using the Jacobi identities associated to gauge and diffeomorphisms transformations

$$
\begin{aligned}
\frac{1}{2} \mathcal{L}_{\mathcal{L}_{\xi} \xi} \phi-\mathcal{L}_{\xi}^{2} \phi & =0 \\
\frac{1}{2}[[c, c], \phi]-[c,[c, \phi]] & =0
\end{aligned}
$$

one obtains the BRST rules for the composite field $\hat{M}(M)$ :

$$
s \hat{M}=-\mathcal{L}_{\xi} \hat{M}-\delta_{c} \hat{M}+\mathcal{L}_{\gamma} M+\delta_{\hat{c}} M
$$

The structure of (4.1) and (4.6) makes it convenient to define an operator $S$ [6], whose action - defined on the matter fields only - is obtained by substracting from $s$ both diffeomorphisms and gauge transformations

$$
S M \equiv s M+\mathcal{L}_{\xi} M+\delta_{c} M
$$

Therefore, by definition,

$$
S M=\hat{M}(M)
$$

From (4.6) it follows that the function of the matter fields

$$
S \hat{M}=\frac{\partial \hat{M}}{\partial M}(M) \hat{M}(M)
$$

must satisfy the relation

$$
S \hat{M}=S^{2} M=\mathcal{L}_{\gamma} M+\delta_{\hat{c}} M
$$

Therefore nilpotency of $s$ on $M$ implies that the operator $S$ obeys the algebra

$$
S^{2}=\mathcal{L}_{\gamma}+\delta_{\hat{c}}
$$

Let us remark that (4.9) and (4.10) represent functional differential equations for the composites $\hat{M}(M)$. When imposing nilpotency of $s$ on $\hat{M}$ we obtain instead

$$
S^{2} \hat{M}=\mathcal{L}_{\gamma} \hat{M}+\delta_{\hat{c}} \hat{M}+\mathcal{L}_{S \gamma} M+\delta_{S \hat{c}} M
$$

We must therefore require

$$
\begin{aligned}
S \gamma^{\mu} & =0 \\
S \hat{c} & =0
\end{aligned}
$$

The BRST equations (4.13) and (4.10) constitute a set of functional equations for the all the composites $-\gamma^{\mu}, \hat{c}$ and $\hat{M}$. Constructing a supergravity theory amounts, in essence, in solving such functional equations. 
We observed already in the previous section that the universal composite defined in (2.3) does indeed satisfy the first of eqs. (4.13). Let us work out the constraints on the form of $\hat{c}$ which follow from the second equation in (4.13). The nilpotency of $s$ on the gauge symmetry ghost $c$ gives

$$
\begin{aligned}
0=s^{2} c= & {\left[c,-\frac{1}{2}[c, c]-\mathcal{L}_{\xi} c+\hat{c}\right]-\mathcal{L}_{-\frac{1}{2} \mathcal{L}_{\xi} \xi^{\mu}+\gamma^{\mu}} c+} \\
& +\mathcal{L}_{\xi}\left(-\frac{1}{2}[c, c]-\mathcal{L}_{\xi} c+\hat{c}\right)+s \hat{c} \\
= & -\left[c, \mathcal{L}_{\xi} c\right]+[c, \hat{c}]+\frac{1}{2} \mathcal{L}_{\mathcal{L}_{\xi} \xi^{\mu}} c-\mathcal{L}_{\gamma} c+ \\
& -\left[\mathcal{L}_{\xi} c, c\right]-\mathcal{L}_{\xi}^{2} c+\mathcal{L}_{\xi} \hat{c}+s \hat{c} \\
= & {[c, \hat{c}]-\mathcal{L}_{\gamma} c+\mathcal{L}_{\xi} \hat{c}+s \hat{c} }
\end{aligned}
$$

where we again used (4.5). Hence we obtain

$$
s \hat{c}=-\mathcal{L}_{\xi} \hat{c}-[c, \hat{c}]+\mathcal{L}_{\gamma} c
$$

On the other hand, since $\hat{c}$ is a composite satisfying the second equation in (4.13), it must be that

$$
0=S \hat{c}=s \hat{c}+\mathcal{L}_{\xi} \hat{c}+\delta_{c} \hat{c}
$$

Comparing (4.15) with (4.16) one deduces the transformation rules of the functional $\hat{c}$ under bosonic gauge symmetry

$$
\delta_{c} \hat{c}=-\mathcal{L}_{\gamma} c+[c, \hat{c}]
$$

In other words, $\hat{c}$ does not transform homogeneously under gauge transformations. It must then have the general form

$$
\hat{c}=i_{\gamma}(A)+\phi
$$

where $A$ is the gauge field 1-form associated to the algebra of the bosonic YM gauge invariances (which include the local Lorentz transformations) and $\phi$ is a composite fields with values in the adjoint of the YM Lie algebra. $\phi$ transforms homogeneously under gauge transformations.

Summarizing, the algebra satisfied by $S$ is

$$
S^{2}=\mathcal{L}_{\gamma}+\delta_{i_{\gamma}(A)+\phi}
$$

The consistency condition $S \hat{c}=0$ translates into the equation

$$
S \phi=i_{\gamma}(S A)
$$

The composite $S A=\hat{A}$ is the topological gaugino, which will be denoted by $\lambda$ :

$$
S A=\lambda
$$


$A$ and $\lambda$ sit into a multiplet with values in the adjoint of the gauge algebra,

$$
\begin{aligned}
S A & =\lambda \\
S \lambda & =i_{\gamma}(F)-D \phi \\
S \phi & =i_{\gamma}(\lambda)
\end{aligned}
$$

These supergravity BRST transformation rules coincide with the BRST rules of topological YM coupled to topological gravity that we wrote in (3.4). Both the topological gaugino $\lambda$ of ghost number +1 and the topological Yang-Mills superghost $\phi$ of ghost number +2 are composite fields in terms of the supergravity fields. This composite topological multiplet represents the universal topological sector which sits inside generic supergravity.

To give a concrete example, let us consider the $d=4, N=1$ "new minimal" supergravity. ${ }^{7}$ The local bosonic YM simmetries of this theory are local Lorentz transformations and local $\mathrm{U}(1)_{R}$ R-symmetry. Let $c$ and $c^{a b}$ be the corresponding anti-commuting ghosts, with $a, b=1, \ldots 4$. The bosonic local simmetries act on the commuting Majorana spinorial ghost $\zeta$ as follows

$$
\delta_{c} \zeta=\left(\frac{i}{2} c \Gamma_{5}+\frac{i}{4} c^{a b} \sigma_{a b}\right) \zeta
$$

Let $\omega^{a b}=d x^{\mu} \omega_{\mu}^{a b}$ be the spin-connection and $A^{(R)}=d x^{\mu} A_{\mu}^{(R)}$ the $\mathrm{U}(1)_{R}$ gauge field. The 1 -form connection with values in the total bosonic YM Lie algebra is

$$
A=-\left(\frac{i}{2} A^{(R)} \Gamma_{5}+\frac{i}{4} \omega^{a b} \sigma_{a b}\right)
$$

The BRST transformations of the Majorana gravitino field $\psi=d x^{\mu} \psi_{\mu}$ take the form

$$
S \psi=-\left(d+A^{+}\right) \zeta=-D^{+} \zeta
$$

where

$$
A^{+}=A+e^{a}\left(\frac{i}{2} \Gamma_{5} H_{a}-\frac{i}{4} \epsilon_{a b c d} \sigma^{c d} H^{b}\right)
$$

is a 1-form with values in the total bosonic gauge Lie algebra, $H_{\mu} d x^{\mu}$ is an auxiliary 1-form field, ${ }^{8}$ and

$$
H_{a}=e_{a}^{\mu} H_{\mu}
$$

The BRST transformations of $H_{a}$ are

$$
S H_{a}=-\frac{i}{8} \epsilon_{a b c d} \bar{\zeta} \Gamma^{b} \psi^{c d}
$$

where $\psi_{a b} \equiv e_{a}^{\mu} e_{b}^{\nu} \psi_{\mu \nu}=e_{a}^{\mu} e_{b}^{\nu}\left(D_{\mu}^{+} \psi_{\nu}-D_{\nu}^{+} \psi_{\mu}\right)$.

\footnotetext{
${ }^{7}$ In the following paragraphs of this section we consider the Minkowskian theory and use conventions and notations of [15]. In particular the normalization of the commuting ghosts and gravitinos differ by those used in the rest of our paper.

${ }^{8}$ The auxiliary field $H_{a}$ is constrained to have zero divergence, up to fermionic terms: more precisely its Hodge dual $\star H$ satisfy: $\star H=d B+\frac{i}{8} \bar{\psi} \Gamma \psi$ where $B$ is a 2 -form.
} 
Recalling the Fierz identity valid for Majorana spinors in four dimensions

$$
\bar{\zeta} \Gamma^{a} \zeta \Gamma_{a} \zeta=0
$$

one verifies that $S$ satisfies the algebra (4.19) with

$$
\phi=-i_{\gamma}\left(e^{a}\right)\left(\frac{3 i}{2} \Gamma_{5} H_{a}+\frac{i}{4} \epsilon_{a b c d} \sigma^{c d} H^{b}\right)
$$

Thus, the composite superghost $\phi$ for $N=1 d=4$ supergravity is $i_{\gamma}$-trivial

$$
\phi=-i_{\gamma}(\Delta) \quad \Delta \equiv \frac{3 i}{2} \Gamma_{5} H+\frac{i}{4} \epsilon_{a b c d} \sigma^{c d} e^{a} H^{b}
$$

When $\phi$ is $i_{\gamma}$-exact, one can introduce a connection

$$
A^{-} \equiv A-\Delta
$$

whose associated composite gaugino is

$$
\lambda^{-} \equiv S A^{-}=\lambda-S \Delta
$$

with

$$
i_{\gamma}\left(\lambda^{-}\right)=0
$$

From the algebra (4.19) we obtain

$$
\begin{aligned}
S \lambda^{-} & =i_{\gamma}(F)-D \phi-\mathcal{L}_{\gamma} \Delta-\left[i_{\gamma}\left(A^{-}\right), \Delta\right]= \\
& =i_{\gamma}\left(F^{-}\right)
\end{aligned}
$$

where

$$
F^{-} \equiv d A^{-}+A^{-2}
$$

is the curvature of the connection $A^{-}$.

We see therefore that when $\phi$ is $i_{\gamma}$-trivial, there exists a connection $A^{-}$and a corresponding topological multiplet $\mathbb{F}^{-}=F^{-}+\lambda^{-}+\phi^{-}$, with vanishing ghost number 2 component, $\phi^{-}=0$. This is a special feature of $N=1 d=4$ supergravity: we will see in the next sections that there exist supergravity models, with extended supersymmetry, for which $\phi$ is not $i_{\gamma}$-exact.

\section{The universal cohomological equations for supersymmetric backgrounds}

Bosonic supergravity configurations which are invariant under supersymmetry trasformations define classical backgrounds to which supersymmmetric quantum field theories (SQFTs) can be coupled. SQFT coupled to such backgrounds are localizable, which means that their partition function is one-loop exact. Supergravity bosonic backgrounds invariant under supersymmetry are identified by generalized convariantly constant spinor equations for the supergravity ghosts $\zeta^{i}$, which are obtained by setting to zero the supersymmetric variations of the spinorial fermionic fields - gravitinos and gauginos of supergravity. 
The results of the previous section show that localizable supergravity backgrounds satisfy universal equations obtained by setting to zero the BRST variations of the composite topological gravitinos and gauginos defined in (2.7) and (4.22). Vanishing of the BRST variation of the composite topological gravitino leads to

$$
D^{\mu} \gamma^{\nu}+D^{\nu} \gamma^{\mu}=0
$$

This equation says that a necessary condition for localization is that the composite vector ghost bilinear (2.3) be a Killing vector of the space-time metric $g^{\mu \nu}$, a condition which is well-known in the localization literature.

The vanishing of the BRST variation of the composite topological gauginos leads instead to the equation

$$
D \phi-i_{\gamma}(F)=0
$$

where $\gamma^{\mu}$ is the Killing vector in eq. (5.1) and $\phi$ is the bilinear of supergravity ghosts which we defined in the previous sections and which characterizes the supergravity BRST algebra (4.19).

The topological equations (5.1)-(5.2) for the generalized Killing spinors $\zeta^{i}$ are universal, in the sense that they take the same form in any dimensions and for any supergravity model, unlike the generalized Killing spinor equations.

It should be emphasized that equations (5.2) are obtained by setting to zero the supergravity BRST variation of a specific (composite) fermion - the topological gaugino $\lambda$. These equations therefore do not, in general, completely characterize the localization locus. There might be more independent equations valid on the localization locus, obtained by setting to zero the BRST variation of other (composite) fermions. For example, for the $N=1 d=4$ new minimal supergravity, on top of equation (5.2), associated to the topological multiplet of the curvature $F$, setting to zero the BRST variation of the gaugino $\lambda^{-}$ in (4.33), one obtains the equation

$$
S \lambda^{-}=i_{\gamma}\left(F^{-}\right)=0
$$

valid for supersymmetric backgrounds. Coming back to the general case, let us remark that in equation (5.2), $F$ and $\phi$ take values in the adjoint representation of the local bosonic gauge symmetries. Therefore eqs. (5.1) split into two separate sets of equations with the same form: one associated to local Lorentz symmetry and the other with additional local YM symmetries.

When either of these symmetries are not abelian eqs. (5.1) are not gauge invariant: their gauge invariant content is captured by the equations satisfied by the generalized Chern classes (3.12):

$$
\left(d-i_{\gamma}\right) c_{n}(\mathbb{F})=0
$$

where

$$
c_{n}=\operatorname{Tr}\left[F^{n}+n F^{n-1} \phi+\cdots+\phi^{n}\right] \quad n=1,2, \ldots
$$

and $\gamma^{\mu}$ is the Killing vector satisfying (5.1). 
We see therefore that the generalized Chern classes evaluated for localizing backgrounds are closed under the coboundary operator

$$
\mathcal{D}_{\gamma}=d-i_{\gamma} \quad \mathcal{D}_{\gamma}^{2}=-\mathcal{L}_{\gamma}
$$

associated to the de Rham cohomology of forms on space-time, equivariant with respect to the action of the Killing vector $\gamma^{\mu}$. We will call the cohomology relative to the coboundary operator $\mathcal{D}_{\gamma}$ the $\gamma$-equivariant (polyform) cohomology.

The $\gamma$-equivariant classes defined by the $c_{n}$ 's are invariants of the localization backgrounds: they are the same for backgrounds which are equivalent under local BRST transformations of supergravity. In other words the classes associated to the $c_{n}$ 's are functions of the moduli of the space of inequivalent localizable backgrounds.

On the other hand it is possible that inequivalent localizable backgrounds give rise to $c_{n}$ 's which are different representatives of the same $\gamma$-equivariant class. In the next section, we will consider more independent, gauge invariant, composite fermions which can be defined for certain supergravity models. Setting to zero their BRST variations one obtains additional topological equations satisfied by supersymmetric backgrounds. In section 7 we will show that these equations allow for a finer classification of the inequivalent localizable backgrounds.

\section{The gauge invariant ghost bilinears}

We have seen in section 4 that for any supergravity theory there exists a scalar ghost bilinear $\phi$ of ghost number +2 with values in the Lie algebra of the bosonic gauge symmetries which characterizes the BRST algebra (4.19). The ghost bilinear $\phi$ is in general a functional of both the supergravity ghosts $\zeta^{i}$ and the bosonic fields of ghost number 0 sitting in the supergravity multiplet. The key property of $\phi$, which can be read off eq. (4.22), is that its BRST variation is $i_{\gamma}$-exact:

$$
S \phi=i_{\gamma}(\lambda)
$$

This property ensures that, on the localization locus, $\phi$ satisfies the topological equation (5.2).

Let us recall that the BRST variation of the supergravity ghosts $\zeta^{i}$ is also $i_{\gamma}$-exact

$$
S \zeta^{i}=i_{\gamma}\left(\psi^{i}\right)
$$

It follows that scalar and gauge invariant ghost bilinears which are indepedent of extra bosonic fields automatically satisfy (6.1). Hence, they give rise to cohomological equations of the form (5.2). We will consider in this paper two supergravity models for which ghost bilinears of this sort can be constructed.

The first one is $N=(2,2)$ supergravity in $d=2$, where the gauge symmetry group is $\mathrm{SO}(2)_{R}$. In this case it is convenient to collect the two Majorana ghosts $\zeta^{i}$, with $i=1,2$, into one single Dirac ghost $\zeta$ on which the gauge group $\mathrm{SO}(2)_{R} \sim \mathrm{U}(1)_{R}$ acts by multiplication by a real phase. ${ }^{9}$ Then the two scalar bilinears

$$
\varphi_{1}=\bar{\zeta} \zeta \quad \varphi_{2}=\bar{\zeta} \Gamma_{3} \zeta
$$

\footnotetext{
${ }^{9}$ The following discussion is valid for both Minkowski and Euclidean signature.
} 
are gauge invariant and thus $S$-invariant modulo $i_{\gamma}$ :

$$
S \varphi_{i}=i_{\gamma}\left(\lambda_{i}\right) \quad i=1,2
$$

where

$$
\lambda_{1} \equiv \bar{\psi} \zeta+\bar{\zeta} \psi \quad \lambda_{2} \equiv \bar{\psi} \Gamma_{3} \zeta+\bar{\zeta} \Gamma_{3} \psi
$$

It follows from the BRST algebra (4.19) that the generalized forms

$$
\mathbb{H}_{i} \equiv \varphi_{i}+\lambda_{i}+\hat{H}_{i}^{(2)}
$$

satisfy

$$
\delta \mathbb{H}_{i}=\left(S+d-i_{\gamma}\right) \mathbb{H}_{i}=0
$$

The 2-forms $\hat{H}_{i}^{(2)}$ write

$$
\hat{H}_{1}^{(2)}=\bar{\psi} \psi+H_{1}^{(2)} \quad \hat{H}_{2}^{(2)}=\bar{\psi} \Gamma_{3} \psi+H_{2}^{(2)}
$$

where $H_{i}^{(2)}$, with $i=1,2$, are the graphiphoton field strengths.

As explained in the previous section, on the localization locus the following cohomological equations hold

$$
d \varphi_{i}-i_{\gamma}\left(H_{i}^{(2)}\right)=0
$$

Scalar ghost bilinears of the same kind can be constructed also for $N=2 d=4$ supergravity in which the R-symmetry $\mathrm{SU}(2)_{R}$ is gauged. In this case we can take the commuting supersymmetry ghosts to be two-components spinors $\zeta_{\alpha}^{i}$ where $\alpha=1,2$ is the Lorentz spinorial index and $i=1,2$ is the index of the fundamental representation of the gauge group $\mathrm{SU}(2)_{R}$, together with their conjugate $\bar{\zeta}_{i}^{\dot{\alpha}}$. Two independent scalar and $\mathrm{SU}(2)_{R}$-invariant ghost bilinears are

$$
\begin{aligned}
& \varphi=\epsilon^{\alpha \beta} \epsilon_{i j} \zeta_{\alpha}^{i} \zeta_{\beta}^{j} \\
& \bar{\varphi}=\epsilon_{\dot{\alpha} \dot{\beta}} \epsilon^{i j} \bar{\zeta}_{i}^{\dot{\alpha}} \bar{\zeta}_{j}^{\dot{\beta}}
\end{aligned}
$$

Again, thanks to (6.2), both $\varphi$ and $\bar{\varphi}$ are $S$-invariant modulo $i_{\gamma}$

$$
\begin{aligned}
& S \varphi=i_{\gamma}\left(\epsilon^{\alpha \beta} \epsilon_{i j} \psi_{\alpha}^{i} \zeta_{\beta}^{j}+\epsilon^{\alpha \beta} \epsilon_{i j} \zeta_{\alpha}^{i} \psi_{\beta}^{j}\right) \equiv i_{\gamma}(\Lambda) \\
& S \bar{\varphi}=i_{\gamma}\left(\epsilon_{\dot{\alpha} \dot{\beta}} \epsilon^{i j} \bar{\psi}_{i}^{\dot{\alpha}} \bar{\zeta}_{j}^{\dot{\beta}}+\epsilon_{\dot{\alpha} \dot{\beta}} \epsilon^{i j} \bar{\zeta}_{i}^{\dot{\alpha}} \bar{\psi}_{j}^{\dot{\beta}}\right) \equiv i_{\gamma}(\bar{\Lambda})
\end{aligned}
$$

where $\psi_{\alpha}^{i}$ and $\bar{\psi}_{i}^{\dot{\alpha}}$ are the gravitinos. Assuming the triviality of the coboundary operator $i_{\gamma}$, the algebra (4.19) ensures that $\varphi$ and $\bar{\varphi}$ sit in abelian topological gauge multiplets

$$
\begin{aligned}
& \mathbb{T} \equiv \varphi+\Lambda+T^{(2)} \\
& \overline{\mathbb{T}} \equiv \bar{\varphi}+\bar{\Lambda}+\bar{T}^{(2)}
\end{aligned}
$$

satisfying

$$
\left(S+d-i_{\gamma}\right) \mathbb{T}=0 \quad\left(S+d-i_{\gamma}\right) \overline{\mathbb{T}}=0
$$


Obtaining the 2-forms $T^{(2)}$ and $\bar{T}^{(2)}$ as functionals of the fields of the supergravity multiplets requires the knowledge of the off-shell BRST transformations of $N=2 d=4$ Poincaré supergravity with gauge group $\mathrm{SU}(2)_{R}$. Since these do not seem to be readily available in the literature we will present this calculation elsewhere. At any rate one can anticipate that the following cohomological $\gamma$-equivariant equations will hold on the localization locus of $N=2, d=4$ supergravity

$$
d \varphi-i_{\gamma}\left(T^{(2)}\right)=0 \quad d \bar{\varphi}-i_{\gamma}\left(\bar{T}^{(2)}\right)=0
$$

We expect these equations to play a central role in understanding the space of localizing backgrounds of $N=2 d=4$ Poincaré supergravity.

\section{An example: $d=2 N=(2,2)$ supergravity}

In this section we will work out the details of the topological structure of $N=(2,2)$ supergravity in $d=2$ with Euclidean signature. ${ }^{10}$ The moduli space of localization backgrounds for this supergravity theory has been fully analysed and described in [4], extending the results of [17]. In the following we will see how the analysis of [4] fits into the framework developed in this paper.

The ghost bilinear $\phi$, characterizing the BRST algebra (4.19) of $N=(2,2)$ supergravity in $d=2$, has the general form

$$
\phi=\phi_{R}(-i \mathbb{I})+\phi_{\text {Lor }}\left(\frac{i}{2} \Gamma_{3}\right)
$$

where $-i \mathbb{I}$ is the generator of the vectorial $\mathrm{U}(1)_{R}$ gauge transformations on Dirac spinors, $\frac{i}{2} \Gamma_{3}$ is the generator of the local Lorentz transformations, $\phi_{R}$ is the scalar ghost bilinear associated to the $\mathrm{U}(1)_{R}$ gauge symmetry and $\phi_{\text {Lor }}$ the one relative to the Lorentz local transformations. The BRST transformations of the gravitino Dirac field $\psi=d x^{\mu} \psi_{\mu}$ write

$$
S \psi=-D \zeta-\frac{i}{2} H_{2} d x^{\mu} \Gamma_{\mu} \zeta-\frac{i}{2} H_{1} d x^{\mu} \Gamma_{3} \Gamma_{\mu} \zeta,
$$

where

$$
D \zeta \equiv d x^{\mu}\left(\partial_{\mu}+\frac{i}{2} \omega_{\mu} \Gamma_{3}-i A_{\mu}\right) \zeta
$$

and $H_{i}=\star H_{i}^{(2)}$, with $i=1,2$, are the scalars dual to the field strengths of the two graviphotons. Taking into account the Fierz identity

$$
\Gamma_{\mu} \zeta \bar{\zeta} \Gamma^{\mu} \zeta=\left(\varphi_{1}-\varphi_{2} \Gamma_{3}\right) \zeta
$$

one derives from $S^{2} \zeta$ the values for the ghost bilinears $\phi_{R}$ and $\phi_{\text {Lor }}$

$$
\begin{aligned}
\phi_{\text {Lor }} & =\mathbb{R}^{(0)}=-\eta^{i j} \varphi_{i} H_{j} \\
\phi_{R} & =\mathbb{F}_{R}^{(0)}=\frac{1}{2} \epsilon^{i j} \varphi_{i} H_{j}
\end{aligned}
$$

\footnotetext{
${ }^{10}$ For a description of $N=(2,2)$ supergravity in two dimensions see, for example, [16].
} 
where $\eta^{i j}$ is the Lorentzian metric $\eta^{11}=-\eta^{22}=1$ and $\epsilon^{i j}$ is the Levi Civita tensor in 2 dimensions. The universal topological equations (5.2) for supersymmetric backgrounds read

$$
\begin{array}{r}
d \phi_{\text {Lor }}-i_{\gamma}\left(R^{(2)}\right)=0 \\
d \phi_{R}-i_{\gamma}\left(\mathcal{F}_{R}^{(2)}\right)=0
\end{array}
$$

These equations mean that the polyforms of degree 2 associated to the curvature and the field strength of the $\mathrm{U}(1)_{R}$ gauge field

$$
\begin{aligned}
\mathbb{R} & =\phi_{\text {Lor }}+R^{(2)} \\
\mathbb{F}_{R} & =\phi_{R}+\mathcal{F}_{R}^{(2)}
\end{aligned}
$$

are $\gamma$-equivariantly closed:

$$
\mathcal{D}_{\gamma} \mathbb{R}=0 \quad \mathcal{D}_{\gamma} \mathbb{F}=0
$$

We explained in section 6 that, for $N=(2,2) d=2$ supergravity, one can construct, starting from the gauge invariant ghost bilinears (6.3), two more equivariant forms $\mathbb{H}_{i}$ of degree 2 , which satisfy the $\gamma$-equivariant cohomology equations (6.9)

$$
\mathcal{D}_{\gamma} \mathbb{H}_{i}=0
$$

on the localization locus.

The relation (7.5) between the scalar components of the equivariantly closed forms $\mathbb{H}_{i}$, $\mathbb{R}$ and $\mathbb{F}_{R}$ can be extended to the following relations between polyforms

$$
\begin{aligned}
\mathbb{F}_{R} & =\frac{1}{2} \epsilon^{i j} \mathbb{H}_{i} L\left(\mathbb{H}_{j}\right) \\
\mathbb{R} & =-\eta^{i j} \mathbb{H}_{i} L\left(\mathbb{H}_{j}\right)
\end{aligned}
$$

where the equivariant form $L\left(\mathbb{H}_{j}\right)$ is defined in eq. (A.8) of the appendix A. These relations show that the universal equations for the curvatures (7.8) are in fact consequence of the equations for the gauge invariant ghost bilinears (7.9). They also connect the cohomology classes of the $\mathbb{H}_{i}$ with those of $\mathbb{R}$ and $\mathbb{F}_{R}$. To see this, let us introduce one more equivariantly closed polyform

$$
\hat{\mathbb{R}}=-\frac{1}{2} \eta^{i j} \mathbb{H}_{i} \mathbb{H}_{j}=-\frac{1}{2}\left(\gamma^{2}+k d \gamma^{2}\right)
$$

where we used the Fierz identity $\varphi_{1}^{2}-\varphi_{2}^{2}=\gamma^{2}$. Note that, since $L$ is a derivative, ${ }^{11}$ one has

$$
\mathbb{R}=L(\hat{\mathbb{R}})
$$

It follows from (7.11) that

$$
\hat{\mathbb{R}}\left(p_{ \pm}\right)=\mathbb{H}_{1}\left(p_{ \pm}\right) \mathbb{H}_{1}\left(p_{ \pm}\right)-\mathbb{H}_{2}\left(p_{ \pm}\right) \mathbb{H}_{2}\left(p_{ \pm}\right)=0
$$

Hence $\hat{\mathbb{R}}$ is cohomologically trivial ${ }^{12}$ and

$$
\left|\mathbb{H}_{1}\left(p_{ \pm}\right)\right|=\left|\mathbb{H}_{2}\left(p_{ \pm}\right)\right|
$$

\footnotetext{
${ }^{11}$ We show this in appendix A.

${ }^{12}$ As mentioned in the appendix $\mathrm{A}, \hat{\mathbb{R}}$ and $\mathbb{R}=L(\hat{\mathbb{R}})$ do not need to be cohomologous.
} 
Let us therefore put

$$
\mathbb{H}_{1}\left(p_{ \pm}\right)=\sigma_{ \pm} \mathbb{H}_{2}\left(p_{ \pm}\right)
$$

where $\sigma_{ \pm}$is the relative sign between $\mathbb{H}_{1}\left(p_{ \pm}\right)$and $\mathbb{H}_{2}\left(p_{ \pm}\right)$. Moreover, from the second of eqs. (7.10) we obtain

$$
L\left(\mathbb{H}_{1}\right)\left(p_{ \pm}\right)=-\frac{\mathbb{R}\left(p_{ \pm}\right)}{\mathbb{H}_{1}\left(p_{ \pm}\right)}+\frac{\mathbb{H}_{2}\left(p_{ \pm}\right)}{\mathbb{H}_{1}\left(p_{ \pm}\right)} L\left(\mathbb{H}_{2}\left(p_{ \pm}\right)\right)
$$

Plugging this inside the first of eqs. (7.10) one arrives to: ${ }^{13}$

$$
\begin{aligned}
\mathbb{F}_{R}\left(p_{ \pm}\right) & =\frac{1}{2}\left(\mathbb{H}_{1}\left(p_{ \pm}\right) L\left(\mathbb{H}_{2}\right)\left(p_{ \pm}\right)-\mathbb{H}_{2}\left(p_{ \pm}\right) L\left(\mathbb{H}_{1}\right)\left(p_{ \pm}\right)\right)= \\
& =\frac{1}{2} \frac{\mathbb{H}_{2}\left(p_{ \pm}\right)}{\mathbb{H}_{1}\left(p_{ \pm}\right)} \mathbb{R}\left(p_{ \pm}\right)=\frac{1}{2} \sigma_{ \pm} \mathbb{R}\left(p_{ \pm}\right)
\end{aligned}
$$

This equivariant cohomology identity — valid for any localizing background — neatly generalizes the equation $F^{(2)}= \pm \frac{1}{2} R^{(2)}$ for ordinary 2-form curvatures which was found long ago to define the topologically twisted $N=2$ models.

In particular eqs. (7.17) together with (A.18) show that the $\gamma$-equivariant cohomology classes of both the curvatures polyforms $\mathbb{F}_{R}$ and $\mathbb{R}$ are (semi)-integral. This should be contrasted with the cohomology classes of the $\mathbb{H}_{i}$ 's which, as shown in [4], depend on a continuous parameter labelling inequivalent localization backgrounds. We believe that this phenomenon is a general property of the $\gamma$-equivariant curvature polyforms of supergravity, although we do not have yet a general proof of it.

\section{Conclusions}

In this paper we showed that a topological structure sits inside supergravity. We did this by writing the BRST algebra of supergravity in the form

$$
S^{2}=\mathcal{L}_{\gamma}+\delta_{i_{\gamma}(A)+\phi}
$$

where $S$ is the supergravity BRST operator - equivariant with respect both gauge transformations and diffeomorphisms $-\mathcal{L}_{\gamma}$ is the Lie derivative along the vector $\gamma \equiv \gamma^{\mu} \frac{\partial}{\partial x^{\mu}}$ and $\delta_{c}$ denotes a gauge transformation with parameter $c$. The two fields $\gamma$ and $\phi$ are bilinears of the commuting supersymmetry ghosts $\zeta^{i}$, where $i=1, \ldots N$ and $N$ is the number of local supersymmetries. The vector field $\gamma$ is given by

$$
\gamma^{\mu}=-\frac{1}{2} \sum_{i} \bar{\zeta}^{i} \Gamma^{\mu} \zeta^{i}
$$

This expression is universal in the sense that it is valid for any supergravity theory in any dimension. On the other hand, the scalar field $\phi$, which is valued in the adjoint representation of the bosonic YM gauge symmetry group, is a ghost bilinear whose dependence on the bosonic fields of supergravity is non-universal, i.e. it is theory dependent.

\footnotetext{
${ }^{13}$ From this relation we derive in particular that $\int_{S^{2}} \mathcal{F}_{R}^{(2)}=\frac{1}{2}\left(\sigma_{+}+\sigma_{-}\right)$. Therefore, the first Chern class of the gauge field can take values $-1,0,1$ according to the signs $\sigma_{ \pm}$, in agreement with [4] and [17].
} 
Our central observation is that both $\gamma^{\mu}$ and $\phi$ have a topological meaning: they can be identified, respectively, with the superghost of topological gravity and the superghost of a topological Yang-Mills theory whose gauge group is the product of the local Lorentz transformations and the Yang-Mills symmetries of the supergravity theory under consideration.

This identification relies on the fact that the supergravity BRST transformations of the ghost bilinears $\gamma^{\mu}$ and $\phi$ coincide precisely with the BRST rules of topological gravity

$$
\begin{aligned}
S g_{\mu \nu} & =\psi_{\mu \nu} \\
S \psi_{\mu \nu} & =\mathcal{L}_{\gamma} g_{\mu \nu} \\
S \gamma^{\mu} & =0
\end{aligned}
$$

and of topological Yang-Mills coupled to topological gravity:

$$
\begin{aligned}
S A & =\lambda \\
S \lambda & =i_{\gamma}(F)-D \phi \\
S \phi & =i_{\gamma}(\lambda)
\end{aligned}
$$

The BRST variations (8.4) are not familiar in the topological Yang-Mills literature, although they already appeared in [3] in a three-dimensional context. In this paper we wrote them down for any dimension and discussed their geometrical interpretation. We believe that these transformations could be of interest to study, for example, the metric dependence of Donaldson invariants - regardless of the application to supergravity that we explored in this article.

It was observed in [3] and [4] that the conditions for unbroken supersymmetry in certain off-shell supergravities with extended supersymmetry can be recast in terms of cohomological equations. In [3] and [4] these cohomological equations were obtained by setting to zero the BRST variations of fermionic fields of topological gravity coupled to some additional topological gauge multiplets.

In this paper we gave a conceptual explanation of this equivalence, by exploiting the topological structure of supergravity captured by the BRST transformations laws (8.3) and (8.4). By setting to zero the BRST variations of the fermions in (8.3) and (8.4) we obtained equations for supersymmetric backgrounds

$$
\begin{aligned}
\mathcal{L}_{\gamma} g_{\mu \nu} & =0 \\
D \phi-i_{\gamma}(F) & =0
\end{aligned}
$$

These equations lead to the $\gamma$-equivariant cohomology equations (5.4), for the equivariant Chern classes of the supergravity Yang-Mills gauge bundle.

From the same BRST algebra (8.1) we derived additional equations, valid on the localization locus, for certain gauge invariant scalar ghost bilinears $\varphi$. These equations also take the form of $\gamma$-equivariant cohomology equations

$$
d \varphi-i_{\gamma}\left(T^{(2)}\right)=0
$$


The scalar gauge invariant ghost bilinears $\varphi$ can be constructed only for certain extended supergravity with specific Yang-Mills groups. The corresponding bosonic 2-forms $T^{(2)}$ are model dependent. In the last section of this paper we have analyzed in detail supergravity with $N=(2,2)$ in $d=2$ for which two such bilinears $\varphi$ can be constructed. We have seen how the $\gamma$-equivariant cohomological equations (8.5) and (8.6) are related to each other, thus providing an a priori explanation of the results presented in [4].

Another theory for which two gauge invariant scalar bilinears $\varphi$ can be constructed is $N=2 d=4$ supergravity. The study of the cohomological equations (8.6) and (8.5) for this theory might lead to the classification of localizing backgrounds for this theory - a long standing problem to which we hope to come back in the future.

In conclusion, we found two types of "composite" BRST topological multiplets sitting inside supergravity: the first are the curvature multiplets, whose 2-form components are the Lorentz and Yang-Mills curvatures. They transform precisely as topological gravity coupled to topological Yang-Mills. They are universal in the sense that they exist for any supergravity theory. The second kind of composite topological multiplets only exist for certain extended supergravities: in this paper we discussed them in detail for the $N=(2,2) d=2$ supergravity example. We plan to report on other examples in the future. These multiplets are gauge invariant and their 0-form components are scalar bilinears of supergravity commuting ghosts. Their top 2-form components involve auxiliary fields.

For $N=(2,2)$ in $d=2$ supergravity we found compact quadratic relations - ultimately descending from Fierz identities - between these two kinds of topological multiplets: these relations express the curvature composite topological multiplets in terms of the gauge invariant composite multiplets. In a sense this shows that, when they exist, the composite gauge invariant topological multiplets, built upon the scalar superghost bilinears, are more "fundamental" than the curvature multiplets.

It would be important to understand if these relations extend to other models: we already checked that they indeed generalize to $N=4 d=2$ supergravity. We will report about this in a forthcoming paper. It would be of great interest to know if relations of this sort hold also for higher dimensional models, most notably for $N=2 d=4$ supergravity.

It is conceivable that relations between curvatures and gauge invariant topological multiplets - beyond having the application to localization of supersymmetric matter that we focused on in this paper - would also give important informations about the dynamics of supergravity itself. Indeed, when such relations hold, the full space of supersymmetric configurations of supergravity is completely described by the moduli space of the equivariant cohomology associated with the gauge invariant multiplets. One is tempted therefore to speculate that also the quantum fluctuations of the supergravity multiplets around those supersymmetric configurations be described by some effective theory which involves only the gauge invariant topological multiplets.

\section{Acknowledgments}

We thank S. Ferrara, S. Murthy and J. Winding for useful discussions. 
CI thanks the Theory Group of KIAS, Seoul and of LPTHE, of the Sorbonne University and CNRS Paris; DR thanks INFN Genoa and the Physics Department of the University of Genoa for their hospitality and financial support during part of this work.

The work of CI was supported in part by INFN, by Genoa University Research Projects, F.R.A. 2015.

\section{A Equivariant cohomology in dimension 2}

In this appendix we collect some facts regarding equivariant forms of degree 2 in 2 dimensions, which are needed to derive the relations (7.10) between the curvature polyforms of $N=(2,2)$ in $d=2$ supergravity and the gauge invariant polyforms $\mathbb{H}_{i}$, with $i=1,2$.

Degree $2 \gamma$-equivariant forms $\Phi$ are polyforms

$$
\Phi=\Phi^{(2)}+\Phi^{(0)}
$$

with

$$
\mathcal{D}_{\gamma} \Phi=0
$$

where $\mathcal{D}_{\gamma}=d-i_{\gamma}$ is the $\gamma$-equivariant exterior derivative associated to the Killing vector $\gamma^{\mu}$ that we introduced in (5.6). In dimension 2, the 0 -form and the 2 -form component of a $\gamma$-equivariant form of degree 2 are related by

$$
\Phi^{(2)}=k d \Phi^{(0)}
$$

where the 1 -form $k$ is the "inverse" of the Killing vector $\gamma^{\mu 14}$

$$
\begin{aligned}
k & \equiv \frac{g_{\mu \nu} \gamma^{\nu}}{\gamma^{2}} d x^{\mu} \quad \gamma^{2} \equiv g_{\mu \nu} \gamma^{\mu} \gamma^{\nu} \\
i_{\gamma}(k) & =1
\end{aligned}
$$

The 2-form $\Phi^{(2)}$ in (A.3) depends on the choice of a metric $g_{\mu \nu}$. However, different metrics with the same Killing vector $\gamma^{\mu}$ give rise to 1 -forms $k$ which differ by 1 -forms $\delta k$ which are both $d$ and $i_{\gamma}$ closed

$$
i_{\gamma}(\delta k)=d(\delta k)=0
$$

Therefore polyforms with (A.3) corresponding to different metrics are cohomologous

$$
\delta \Phi^{(2)}=d\left(\delta k \Phi^{(0)}\right) \quad \delta \Phi^{(0)}=i_{\gamma}\left(\delta k \Phi^{(0)}\right)=0
$$

Let us also observe that the product of two $\gamma$-equivariant forms of degree 2 in 2 dimensions is again a $\gamma$-equivariant form of degree 2 , i.e. the set of $\gamma$-equivariant forms of degree 2 in 2 dimensions has a ring structure:

$$
\begin{aligned}
\Phi_{1} \Phi_{2} & =\Phi_{1}^{(2)} \Phi_{2}^{(0)}+\Phi_{0}^{(2)} \Phi_{1}^{(0)}+\Phi_{1}^{(0)} \Phi_{2}^{(0)} \\
\mathcal{D}_{\gamma}\left(\Phi_{1} \Phi_{2}\right) & =0
\end{aligned}
$$

\footnotetext{
${ }^{14}$ Since $\gamma^{\mu}$ may have zeros, $k$ may have poles. However, one can show that $\Phi^{(2)}$ defined in (A.3) is regular when $\Phi^{(0)}$ satisfies the equivariance equation (A.2).
} 
Moreover, given a metric, one can introduce a linear operation $L$ which when acting on a $\gamma$-equivariant form of degree 2 gives another $\gamma$-equivariant form of degree 2 :

$$
\begin{aligned}
L(\Phi) & =\star\left[D_{\mu}\left(\frac{1}{\gamma^{2}} D^{\mu} \Phi^{(0)}\right)\right]+\star \Phi^{(2)} \equiv \\
& \equiv \star\left(\Delta_{\gamma} \Phi^{(0)}\right)+\star \Phi^{(2)} \\
\mathcal{D}_{\gamma} L(\Phi) & =0
\end{aligned}
$$

where we introduced the $\gamma$-dependent Laplacian

$$
\Delta_{\gamma} \Phi^{(0)} \equiv D_{\mu}\left(\frac{1}{\gamma^{2}} D^{\mu} \Phi^{(0)}\right)
$$

for 0 -forms ${ }^{15}$. $L$ acts as a derivative with respect to the product (A.7)

$$
L\left(\Phi_{1} \Phi_{2}\right)=L\left(\Phi_{1}\right) \Phi_{2}+\Phi_{1} L\left(\Phi_{2}\right)
$$

It should be kept in mind the $L$ does not map $\gamma$-equivariant forms to cohomologous ones.

Let us observe the cohomology class of a $\gamma$-equivariant form $\Phi$ of degree 2 on the 2dimensional sphere is parametrized by the values of the polyform at the two zeros $p_{ \pm}$of the Killing vector $\gamma^{\mu}$ :

$$
\Phi\left(p_{ \pm}\right)=\Phi^{(0)}\left(p_{ \pm}\right)
$$

The cohomological invariant obtained by evaluating $\Phi$ on the sphere is related to $\Phi\left(p_{ \pm}\right)$ by the localization formula

$$
\int_{S^{2}} \Phi=\int_{S^{2}} \Phi^{(2)}=\Phi^{(0)}\left(p_{+}\right)-\Phi^{(0)}\left(p_{-}\right)
$$

Let us introduce the equivariantly closed polyform

$$
\hat{\mathbb{R}}=-\frac{1}{2} \eta^{i j} \mathbb{H}_{i} \mathbb{H}_{j}=-\frac{1}{2}\left(\gamma^{2}+k d \gamma^{2}\right)
$$

where we used the Fierz identity $\varphi_{1}^{2}-\varphi_{2}^{2}=\gamma^{2}$. Note that

$$
\mathbb{R}=L(\hat{\mathbb{R}})=\phi_{\text {Lor }}+R^{(2)}
$$

From (A.13) one obtain the following expression for the $\gamma$-equivariant extension of curvature 2-form:

$$
\begin{aligned}
\mathbb{R} & =\frac{1}{2} \sqrt{g} \epsilon_{\mu \nu} D^{\mu} \gamma^{\nu}+R^{(2)}= \\
& =i_{\gamma}\left(\star \frac{d \gamma^{2}}{2 \gamma^{2}}\right)-d \star\left(\frac{d \gamma^{2}}{2 \gamma^{2}}\right)
\end{aligned}
$$

\footnotetext{
${ }^{15}$ Since $\gamma^{\mu}$ may in general have zeros, the action of $\Delta_{\gamma}$ on a generic 0 -form is not always well-defined. However it can be shown that $\Delta_{\gamma} \Phi^{(0)}$ is regular when $\Phi^{(0)}$ is the 0 -form component of a $\gamma$-equivariant form of degree 2 .
} 
This equivariant form depends on the metric and the corresponding Killing vector. Under a variation of the metric $\delta g_{\mu \nu}$, keeping the Killing vector fixed, it varies by cohomologically trivial terms

$$
\begin{aligned}
& \delta R^{(2)}=d \delta \omega^{(1)} \quad \delta \phi_{\text {Lor }}=i_{\gamma}\left(\delta \omega^{(1)}\right) \\
& \delta \omega^{(1)}=\delta\left(\star \frac{d \gamma^{2}}{2 \gamma^{2}}\right)
\end{aligned}
$$

In particular

$$
\delta \mathbb{R}\left(p_{ \pm}\right)=0
$$

Therefore the $\gamma$-equivariant cohomology class of $\mathbb{R}$ is independent of the metric and can be computed, for example, using the round metric:

$$
\mathbb{R}\left(p_{ \pm}\right)= \pm 1
$$

Open Access. This article is distributed under the terms of the Creative Commons Attribution License (CC-BY 4.0), which permits any use, distribution and reproduction in any medium, provided the original author(s) and source are credited.

\section{References}

[1] V. Pestun et al., Localization techniques in quantum field theories, J. Phys. A 50 (2017) 440301 [arXiv: 1608.02952] [INSPIRE].

[2] G. Festuccia and N. Seiberg, Rigid Supersymmetric Theories in Curved Superspace, JHEP 06 (2011) 114 [arXiv: 1105.0689] [INSPIRE].

[3] C. Imbimbo and D. Rosa, Topological anomalies for Seifert 3-manifolds, JHEP 07 (2015) 068 [arXiv: 1411.6635] [INSPIRE].

[4] J. Bae, C. Imbimbo, S.-J. Rey and D. Rosa, New Supersymmetric Localizations from Topological Gravity, JHEP 03 (2016) 169 [arXiv:1510.00006] [INSPIRE].

[5] L. Baulieu and M.P. Bellon, p Forms and Supergravity: Gauge Symmetries in Curved Space, Nucl. Phys. B 266 (1986) 75 [inSPIRE].

[6] C. Imbimbo, The Coupling of Chern-Simons Theory to Topological Gravity, Nucl. Phys. B 825 (2010) 366 [arXiv: 0905.4631] [INSPIRE].

[7] C.M. Becchi and C. Imbimbo, Gribov horizon, contact terms and Cech-De Rham cohomology in 2-D topological gravity, Nucl. Phys. B 462 (1996) 571 [hep-th/9510003] [INSPIRE].

[8] L. Baulieu and A. Tanzini, Topological gravity versus supergravity on manifolds with special holonomy, JHEP 03 (2002) 015 [hep-th/0201109] [INSPIRE].

[9] D. Rosa, The coupling of Poisson $\sigma$-models to topological backgrounds, JHEP 12 (2016) 043 [arXiv: 1610.05659] [INSPIRE].

[10] P. Schaller and T. Strobl, Poisson structure induced (topological) field theories, Mod. Phys. Lett. A 9 (1994) 3129 [hep-th/9405110] [INSPIRE].

[11] N. Ikeda, Two-dimensional gravity and nonlinear gauge theory, Annals Phys. 235 (1994) 435 [hep-th/9312059] [INSPIRE]. 
[12] S.K. Donaldson, Irrationality and the h-cobordism conjecture, J. Diff. Geom. 26 (1987) 141 [INSPIRE].

[13] S.K. Donaldson, Polynomial invariants for smooth manifolds, Topology 29 (1990) 257 [INSPIRE].

[14] G.W. Moore and E. Witten, Integration over the u plane in Donaldson theory, Adv. Theor. Math. Phys. 1 (1997) 298 [hep-th/9709193] [INSPIRE].

[15] S. Ferrara and S. Sabharwal, Structure of New Minimal Supergravity, Annals Phys. 189 (1989) 318 [INSPIRE].

[16] S.J. Gates Jr., Y. Hassoun and P. van Nieuwenhuizen, Auxiliary Fields for $d=2, N=4$ Supergravity, Nucl. Phys. B 317 (1989) 302 [InSPIRE].

[17] C. Closset and S. Cremonesi, Comments on $\mathcal{N}=(2,2)$ supersymmetry on two-manifolds, JHEP 07 (2014) 075 [arXiv: 1404.2636] [INSPIRE]. 